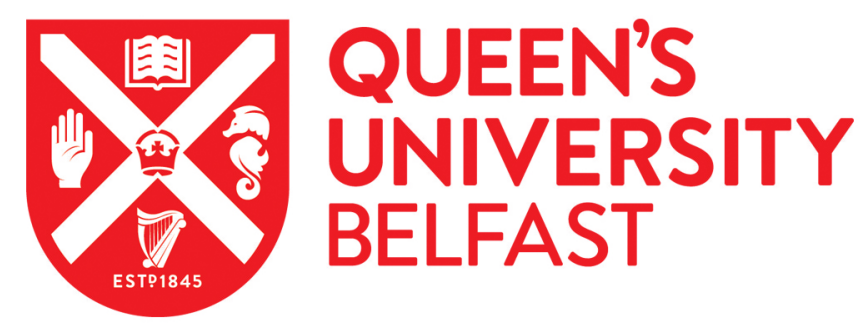

\title{
Model Predictive Control for Smart Grids with Multiple Electric-Vehicle Charging Stations
}

Shi, Y., Tuan, H. D., Savkin, A. A., Duong, T. Q., \& Poor, H. V. (2018). Model Predictive Control for Smart Grids with Multiple Electric-Vehicle Charging Stations. IEEE Transactions on Smart Grid.

https://doi.org/10.1109/TSG.2017.2789333

\section{Published in:}

IEEE Transactions on Smart Grid

\section{Document Version:}

Peer reviewed version

Queen's University Belfast - Research Portal:

Link to publication record in Queen's University Belfast Research Portal

\section{Publisher rights}

(C) 2017 IEEE.

This work is made available online in accordance with the publisher's policies. Please refer to any applicable terms of use of the publisher.

\section{General rights}

Copyright for the publications made accessible via the Queen's University Belfast Research Portal is retained by the author(s) and / or other copyright owners and it is a condition of accessing these publications that users recognise and abide by the legal requirements associated with these rights.

Take down policy

The Research Portal is Queen's institutional repository that provides access to Queen's research output. Every effort has been made to ensure that content in the Research Portal does not infringe any person's rights, or applicable UK laws. If you discover content in the Research Portal that you believe breaches copyright or violates any law, please contact openaccess@qub.ac.uk. 


\title{
Model Predictive Control for Smart Grids with Multiple Electric-Vehicle Charging Stations
}

\author{
Y. Shi, H. D. Tuan, A. V. Savkin, T. Q. Duong and H. V. Poor
}

\begin{abstract}
Next-generation power grids will likely enable concurrent service for residences and plug-in electric vehicles (PEVs). While the residence power demand profile is known and thus can be considered inelastic, the PEVs' power demand is only known after random PEVs' arrivals. PEV charging scheduling aims at minimizing the potential impact of the massive integration of PEVs into power grids to save service costs to customers while power control aims at minimizing the cost of power generation subject to operating constraints and meeting demand. The present paper develops a model predictive control (MPC)based approach to address the joint PEV charging scheduling and power control to minimize both PEV charging cost and energy generation cost in meeting both residence and $P E V$ power demands. Unlike in related works, no assumptions are made about the probability distribution of PEVs' arrivals, the known PEVs' future demand, or the unlimited charging capacity of PEVs. The proposed approach is shown to achieve a globally optimal solution. Numerical results for IEEE benchmark power grids serving Tesla Model S PEVs show the merit of this approach.
\end{abstract}

Index Terms-Smart power grid, plug-in electric vehicles, model predictive control, optimal power flow.

\section{INTRODUCTION}

Electrical vehicles (EVs) have emerged as a promising solution to resolve both the economic and environmental concerns in the transportation industry [1]. Using a smart power grid in concurrently serving residences and charging EVs constitutes one of the most important applications of the smart grid technology. However, the massive integration of plug-in EVs (PEVs) into the grid causes many potential impacts such as voltage deviation, increased load variations and power loss of the grid [2], which requires different strategies for load shifting and energy trading and storage in the grid [3]-[6]. The main difficulty in scheduling of PEV charging to manage the cost and impact of PEV integration is that individual PEVs randomly arrive for charging with their

This work was supported in part by the Australian Research Council's Discovery Projects under Project DP130104617 and DP170103750, in part by the U.K. Royal Academy of Engineering Research Fellowship under Grant RF1415/14/22, in part by the U.K. Engineering and Physical Sciences Research Council (EPSRC) under Grant EP/P019374/1, and in part by the U.S. National Science Foundation under Grants ECCS-1549881.

Ye Shi and Hoang D. Tuan are with the School of Electrical and Data Engineering, University of Technology Sydney, Broadway, NSW 2007, Australia (email: Ye.Shi@student.uts.edu.au, Tuan.Hoang@uts.edu.au)

Andrey V. Savkin is with the School of Electrical Engineering and Telecommunications, The University of New South Wales Sydney, NSW 2052, Australia (email: a.savkin@unsw.edu.au)

Trung Q. Duong is with Queen's University Belfast, Belfast BT7 1NN, UK (email: trung.q.duong@qub.ac.uk)

H. Vincent Poor is with the Department of Electrical Engineering, Princeton University, Princeton, NJ 08544, USA (e-mail: poor@princeton.edu) individual demands on charging load and deadlines, which cannot be known before hand. In other words, the future charging demand of PEVs cannot be known a priori. Many existing works consider a simple smart grid with a single charging station (CS) to exclusively serve PEVs. For instance, [7] sets no charging deadlines for PEVs, whose arrival process follows a probability distribution, while [8] assumes that the future load demand is perfectly known a priori. The future load demand is also assumed to be known in [9] as all PEVs are assumed to arrive at the same time with no charging deadline. It is assumed in [10] that only statistics of demand are known but the PEVs can be fully charged in a single time slot $[10$, (30)]. It should be realized that serving PEVs is typically considered during a 12-hour time period (for instance from 8:00 $\mathrm{pm}$ to 8:00 am), where the integration of a massive number of PEVs has a sizable effect on the power grid, and as such, the length of a time slot is rationally set by 30 minutes or one hour. In other words, the charging scheduling should be considered over a finite horizon of 12-24 time slots, but not over an infinite horizon as considered in [11]. Due to their physical limitations, PEVs are rarely able to be fully charged just during a single time slot.

In this paper, we consider joint PEV charging scheduling and power control to save service costs for PEVs and the power generation costs in meeting both residential and PEV power demands. Such a problem was considered in [12] but only a small number of PEVs and with each CS serving only one PEV, whose power demand is very small compared with the inelastic demand, so that the integration of PEVs into the grid has almost no effect on the grid. Note that the optimal power flow problems posed in [12] cannot be solved exactly by semi-definite programming relaxation (SDR) [13]. Therefore, it is not known if the objective in PEVs charging scheduling is convex and as such, it is not known if its proposed valleyfilling solution is optimal. Larger PEVs' penetration in a few CSs was considered in [14], [15] under the assumption of known arrival and departure times of PEVs. In the present paper, we are interested in more practical scenarios of a massive number of PEVs arriving randomly at different CSs. No assumption on the probability distribution of their arrival is made, so the conventional model predictive control (MPC) [16], [17] is not applicable. Our contribution is to develop a novel MPC-based approach to address this problem.

The rest of the paper is structured as follows. Section II is devoted to the system modeling for this problem and analyzing its computational challenges. An online computational solution using the proposed MPC-based approach is developed in Section III. An off-line computational solution 
is considered in Section IV, which is then compared with the online computational solution in Section $\mathrm{V}$ to show the optimality of the later. Section VI concludes the paper.

Notation. The notation used in this paper is standard. Particularly, $j$ is the imaginary unit, $X^{H}$ is Hermitian transpose of a vector/matrix $X, M \succeq 0$ for a Hermitian symmetric matrix $M$ means that it is positive semi-definite, $\operatorname{rank}(M)$ and $\operatorname{Trace}(M)$ are the rank and trace of a matrix $M$, respectively. $\Re(\cdot)$ and $\Im(\cdot)$ are the real and imaginary parts of a complex quantity, and $a \leq b$ for two complex numbers $a$ and $b$ is componentwise understood, i.e. $\Re(a) \leq \Re(b)$ and $\Im(a) \leq \Im(b)$. The cardinality of a set $\mathcal{C}$ is denoted by $|\mathcal{C}|$.

\section{Problem statement and Computational CHALLENGES}

Consider an electricity grid with a set of buses $\mathcal{N}:=$ $\{1,2, \ldots, N\}$ connected through a set of flow lines $\mathcal{L} \subseteq \mathcal{N} \times \mathcal{N}$, i.e. bus $k$ is connected to bus $m$ if and only if $(k, m) \in \mathcal{L}$. Accordingly, $\mathcal{N}(k)$ is the set of other buses connected to bus $k$. There is a subset $\mathcal{G} \subseteq \mathcal{N}$, whose elements are connected to distributed generators (DGs). Any bus $k \notin \mathcal{G}$ is thus not connected to DGs. Any bus $k \in \mathcal{G}$ also has a function to serve PEVs and in what follow is also referred to CS $k$. By defining $M=|\mathcal{G}|$, there are $M$ CSs in the grid. Denote by $\mathcal{H}_{k}$ the set of those PEVs that arrive at CS $k$. Accordingly, $k_{n}$ is the $n$-th PEV that arrives at CS $k$. Figure 1 provides a general structure of PEV charging in a smart grid system.

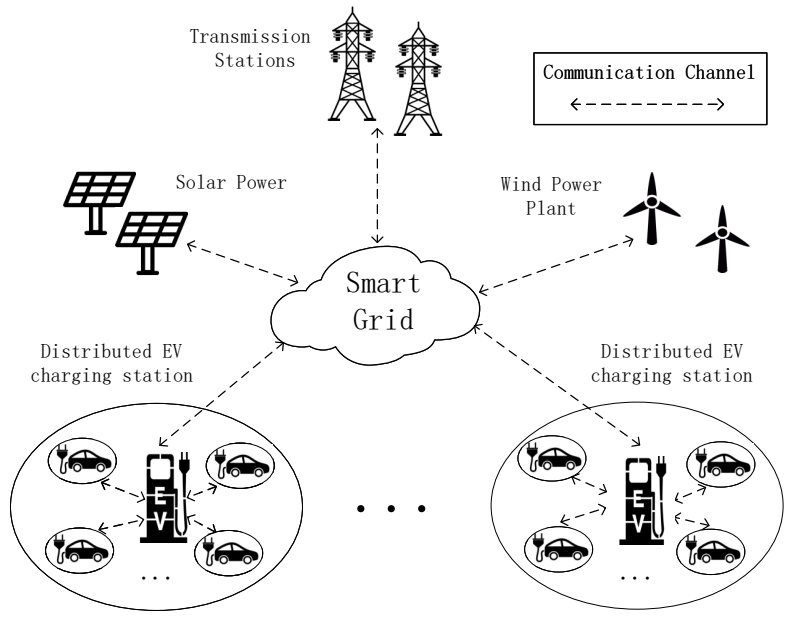

Fig. 1: System architecture of PEV charging in smart grid

The serving time period of the grid is divided into $T$ time slots of length $\delta_{t}$, which usually varies from 30 minutes to an hour. Under the definition $\mathcal{T}:=\{1,2, \ldots, T\}$, PEV $k_{n}$ arrives at $t_{a, k_{n}} \in \mathcal{T}$ and needs to depart at $t_{k_{n}, d} \in \mathcal{T}$. The constraint

$$
t_{k_{n}, d}-t_{k_{n}, a} \leq T_{k_{n}},
$$

expresses the PEV $k_{n}$ 's time demand. Suppose that $C_{k_{n}}$ and $s_{k_{n}}^{0}$ are the battery capacity and initial state of charge (SOC) of PEV $k_{n}$. It must be fully charged by the departure time $t_{k_{n}, d}$, i.e.

$$
\sum_{t^{\prime}=t_{k_{n}, a}}^{t_{k_{n}, d}} u_{h} P_{k_{n}}\left(t^{\prime}\right)=C_{k_{n}}\left(1-s_{k_{n}}^{0}\right),
$$

where $u_{h}$ is the charging efficiency of the battery and $P_{k_{n}}\left(t^{\prime}\right)$ is a decision variable representing the power charging rate of PEV $k_{n} \in \mathcal{H}_{k}$ at time $t^{\prime}$.

Due to the limited capacity of the hardware, the following constraint must be imposed:

$$
0 \leq P_{k_{n}}\left(t^{\prime}\right) \leq \bar{P}_{k_{n}}, t_{k_{n}, a} \leq t^{\prime} \leq t_{k_{n}, d},
$$

for a given $\bar{P}_{k, n}$. For ease of presentation, we set

$$
P_{k_{n}}\left(t^{\prime}\right)=0, t^{\prime} \notin\left[t_{k_{n}, a}, t_{k_{n}, b}\right] .
$$

Let $y_{k m} \in \mathbb{C}$ be the admittance of line $(k, m)$. The current $I_{k}\left(t^{\prime}\right)$ at node $k \in \mathcal{N}$ is,

$$
\begin{aligned}
I_{k}\left(t^{\prime}\right) & =\sum_{m \in \mathcal{N}(k)} I_{k m}\left(t^{\prime}\right) \\
& =\sum_{m \in \mathcal{N}(k)} y_{k m}\left(V_{k}\left(t^{\prime}\right)-V_{m}\left(t^{\prime}\right)\right),
\end{aligned}
$$

where $V_{k}\left(t^{\prime}\right)$ is the complex voltage at bus $k$ during the time slot $t^{\prime}$. For $k \in \mathcal{G}$, the total supply and demand energy is balanced as,

$$
\begin{array}{r}
V_{k}\left(t^{\prime}\right)\left(I_{k}\left(t^{\prime}\right)\right)^{*}=\left(P_{g_{k}}\left(t^{\prime}\right)-\right. \\
\left.P_{l_{k}}\left(t^{\prime}\right)-\sum_{n \in \mathcal{H}_{k}} P_{k_{n}}\left(t^{\prime}\right)\right) \\
+j\left(Q_{g_{k}}\left(t^{\prime}\right)-Q_{l_{k}}\left(t^{\prime}\right)\right),
\end{array}
$$

where $P_{g_{k}}\left(t^{\prime}\right)$ and $Q_{g_{k}}\left(t^{\prime}\right)$ are the real and reactive powers generated by DG $k$, and $P_{l_{k}}\left(t^{\prime}\right)$ and $Q_{l_{k}}\left(t^{\prime}\right)$ are respectively known real and reactive price-inelastic demands at bus $k$ to express the residential power demand. By using the last two equations, we obtain

$$
\begin{array}{r}
V_{k}\left(t^{\prime}\right)\left(\sum_{m \in \mathcal{N}(k)} y_{k m}\left(V_{k}\left(t^{\prime}\right)-V_{m}\left(t^{\prime}\right)\right)\right)^{*}=\left(P_{g_{k}}\left(t^{\prime}\right)\right. \\
\left.-P_{l_{k}}\left(t^{\prime}\right)-\sum_{n \in \mathcal{H}_{k}} P_{k_{n}}\left(t^{\prime}\right)\right)+j\left(Q_{g_{k}}\left(t^{\prime}\right)-Q_{l_{k}}\left(t^{\prime}\right)\right), k \in \mathcal{G} .
\end{array}
$$

Similarly,

$$
\begin{array}{r}
V_{k}\left(t^{\prime}\right)\left(\sum_{m \in \mathcal{N}(k)} y_{k m}\left(V_{k}\left(t^{\prime}\right)-V_{m}\left(t^{\prime}\right)\right)\right)^{*}= \\
-P_{l_{k}}\left(t^{\prime}\right)-j Q_{l_{k}}\left(t^{\prime}\right), k \notin \mathcal{G} .
\end{array}
$$

The next constraints relate to the acceptable range of generated power by the DGs:

$\underline{P}_{g_{k}} \leq P_{g_{k}}\left(t^{\prime}\right) \leq \bar{P}_{g_{k}} \& \underline{Q}_{g_{k}} \leq Q_{g_{k}}\left(t^{\prime}\right) \leq \bar{Q}_{g_{k}}, k \in \mathcal{G}$,

where $\underline{P}_{g_{k}}, \underline{Q}_{g_{k}}$ and $\bar{P}_{g_{k}}, \bar{Q}_{g_{k}}$ are the the lower limit and upper limit of the real generated and reactive generated powers, respectively.

The constraints of voltage are

$$
\begin{aligned}
\underline{V}_{k} & \leq\left|V_{k}\left(t^{\prime}\right)\right| \leq \bar{V}_{k}, \quad k \in \mathcal{N}, \\
\left|\arg \left(V_{k}\left(t^{\prime}\right)\right)-\arg \left(V_{m}\left(t^{\prime}\right)\right)\right| & \leq \theta_{k m}^{\max },(k, m) \in \mathcal{L}, t^{\prime} \in \mathcal{T},
\end{aligned}
$$

where $\underline{V}_{k}$ and $\bar{V}_{k}$ are the lower limit and upper limit of the voltage amplitude, while $\theta_{k, m}^{\max }$ are given to express the voltage phase balance.

The constraints of line capacity are

$$
\begin{array}{r}
\left|V_{k}\left(t^{\prime}\right)\left(V_{k}\left(t^{\prime}\right)^{*}-V_{m}\left(t^{\prime}\right)^{*}\right) y_{k m}^{*}\right| \leq S_{k m}, \\
(k, m) \in \mathcal{L}, t^{\prime} \in \mathcal{T},
\end{array}
$$


where $S_{k m}$ is the upper limit of capacity for line $(k, m)$.

The problem of interest is to minimize both the energy cost to DGs and charging cost for PEVs. Thus, by defining

$$
\begin{gathered}
V\left(t^{\prime}\right)=\left(V_{1}\left(t^{\prime}\right), \ldots, V_{N}\left(t^{\prime}\right)\right), \quad \mathcal{V}=\left\{V\left(t^{\prime}\right)\right\}_{t^{\prime} \in \mathcal{T}}, \\
P_{g}\left(t^{\prime}\right)=\left(P_{g_{1}}\left(t^{\prime}\right), \ldots, P_{g_{M}}\left(t^{\prime}\right)\right), \\
Q_{g}\left(t^{\prime}\right)=\left(Q_{g_{1}}\left(t^{\prime}\right), \ldots, Q_{g_{M}}\left(t^{\prime}\right)\right), \\
R\left(t^{\prime}\right)=\left\{P_{g}\left(t^{\prime}\right), Q_{g}\left(t^{\prime}\right)\right\}, \quad \mathcal{R}=\left\{R\left(t^{\prime}\right)\right\}_{t^{\prime} \in \mathcal{T}}, \\
\mathcal{P}^{P E V}=\left\{P^{P E V}\left(t^{\prime}\right)\right\}_{t^{\prime} \in \mathcal{T}}, \\
P^{P E V}\left(t^{\prime}\right)=\left\{P_{k_{n}}\left(t^{\prime}\right)\right\}_{k_{n} \in \mathcal{H}_{k}, k=1, \ldots, M},
\end{gathered}
$$

the objective function is given by

$$
\begin{aligned}
F\left(\mathcal{R}, \mathcal{P}^{P E V}\right)= & \sum_{t^{\prime} \in \mathcal{T}} \sum_{k \in \mathcal{G}} f\left(P_{g_{k}}\left(t^{\prime}\right)\right) \\
& +\sum_{t^{\prime} \in \mathcal{T}} \sum_{k \in \mathcal{N}} \sum_{n \in \mathcal{H}_{k}} \beta_{t} P_{k_{n}}\left(t^{\prime}\right),
\end{aligned}
$$

where $f\left(P_{g_{k}}\left(t^{\prime}\right)\right)$ is the cost function of real power generation by DGs, which is linear or quadratic in $P_{g_{k}}\left(t^{\prime}\right)$, and $\beta_{t}$ is the known PEV charging price during the time interval $\left(t^{\prime}, t^{\prime}+1\right]$.

The joint PEV charging scheduling and power control is then mathematically formulated as

$$
\min _{\mathcal{V}, \mathcal{R}, \mathcal{P}^{P E V}} F\left(\mathcal{R}, \mathcal{P}^{P E V}\right) \quad \text { s.t. } \quad(2)-(10) .
$$

The above problem (11) is very computationally challenging because the quadratic equality constraints (5) and (6) and nonlinear inequality constraints (8) and (9) constitute nonconvex constraints. Moreover, the arrival time $t_{k_{n}, a}$ of each individual PEV $k_{n}$, its charging demand and its departure time $t_{k_{n}, d}$ are unknown.

\section{MODEL PREDICTIVE CONTROL (MPC)-BASED COMPUTATIONAL SOLUTION}

Considering $\left(R\left(t^{\prime}\right), P^{P E V}\left(t^{\prime}\right)\right)$ and $V\left(t^{\prime}\right)$ as the plant state and control, respectively, equations (5), (6), and (7) provide state behavioral equations [18] with the end constraint (2) while equations (8) and (9) provide control constraints. On the surface, (11) appears to be a control problem over the finite horizon $[1, T]$. However, all equations in (11) are unpredictable beforehand, preventing the application of conventional model predictive control (MPC) [16], [17]. We now follow the idea of [19] to address (11).

The conventional MPC relies on the two key steps at time $t$ : predicting future events and minimizing a reference-based cost function by considering the plant over a short receding horizon $[t, t+T]$. Both these steps cannot be implemented for problem (11) because the PEVs' arrivals cannot be anticipated while there is no reference for PEV charging. Our proposed method, which does not need prediction for PEVs' arrivals or reference for PEV charging is described as follow. At each time $t$ denote by $C(t)$ the set of PEVs that need to be charged. For each $k_{n} \in C(t)$, let $\mathcal{P}_{k_{n}}(t)$ be its remaining demand for charging by the departure time $t_{k_{n}, d}$. Define

$$
\Psi(t)=\max _{k_{n} \in C(t)} t_{k_{n}, d}
$$

At time $t$ we solve the following optimal power flow (OPF) problem over the prediction horizon $[t, \Psi(t)]$ but then take only $V(t), P_{k_{n}}(t), R(t)$ for online updating solution of (11):

$$
\begin{array}{r}
V\left(t^{\prime}\right), R\left(t^{\prime}\right), P_{k_{n}}\left(t^{\prime}\right), t^{\prime} \in[t, \Psi(t)], k_{n} \in C(t) \\
\text { s.t. } \quad(3)-(4),(6)-(10) \quad \text { for } t^{\prime} \in[t, \Psi(t)] \\
V_{k}\left(t^{\prime}\right)\left(\sum_{m \in \mathcal{N}(k)} y_{k m}\left(V_{k}\left(t^{\prime}\right)-V_{m}\left(t^{\prime}\right)\right)\right)^{*}= \\
\left(P_{g_{k}}\left(t^{\prime}\right)-P_{l_{k}}\left(t^{\prime}\right)-\sum_{k_{n} \in C(t)} P_{k_{n}}\left(t^{\prime}\right)\right) \\
+j\left(Q_{g_{k}}\left(t^{\prime}\right)-Q_{l_{k}}\left(t^{\prime}\right)\right), \\
\text { for }\left(t^{\prime}, k\right) \in[t, \Psi(t)] \times \mathcal{G}, \\
\sum_{t_{k_{n}, d}=t} u_{h} P_{k_{n}}\left(t^{\prime}\right)=\mathcal{P}_{k_{n}}(t),
\end{array}
$$

with

$$
F_{[t, \Psi(t)]}:=\sum_{t^{\prime}=t}^{\Psi(t)} \sum_{k \in \mathcal{G}} f\left(P_{g_{k}}\left(t^{\prime}\right)\right)+\sum_{t^{\prime}=t}^{\Psi(t)} \sum_{k_{n} \in C(t)} \beta_{t} P_{k_{n}}\left(t^{\prime}\right) .
$$

One can notice that (13) includes only what is known at the present time $t$. Of course, (13) is a still difficult nonconvex optimization and in the end we need only its solution at $t$, so we propose the following approach in tackling its solution at $t$.

Define the Hermitian symmetric matrix $W\left(t^{\prime}\right)=$ $V\left(t^{\prime}\right) V^{H}\left(t^{\prime}\right) \in \mathbb{C}^{N \times N}$, which must satisfy $W\left(t^{\prime}\right) \succeq 0$ and $\operatorname{rank}\left(W\left(t^{\prime}\right)\right)=1$. By replacing $W_{k m}\left(t^{\prime}\right)=V_{k}\left(t^{\prime}\right) V_{m}^{*}\left(t^{\prime}\right)$, $(k, m) \in \mathcal{N} \times \mathcal{N}$, in (13c)-(13b), we reformulate (13) to the following optimization problem in matrices $W\left(t^{\prime}\right) \in \mathbb{C}^{N \times N}$, $t^{\prime} \in[t, \Psi(t)]:$

$$
\begin{aligned}
& \min _{W\left(t^{\prime}\right), R\left(t^{\prime}\right), P_{k_{n}}\left(t^{\prime}\right), t^{\prime} \in[t, \Psi(t)], k_{n} \in C(t)} F_{[t, \Psi(t)]}(14 \mathrm{a}) \\
& \text { s.t. } \quad(3)-(4),(7) \text { for } t^{\prime} \in[t, \Psi(t)],(14 \mathrm{~b}) \\
& \sum_{m \in \mathcal{N}(k)}\left(W_{k k}\left(t^{\prime}\right)-W_{k m}\left(t^{\prime}\right)\right) y_{k m}^{*}=\left(P_{g_{k}}\left(t^{\prime}\right)-P_{l_{k}}\left(t^{\prime}\right)\right. \\
& \left.-\sum_{k_{n} \in C(t)} P_{k_{n}}\left(t^{\prime}\right)\right)+j\left(Q_{g_{k}}\left(t^{\prime}\right)-Q_{l_{k}}\left(t^{\prime}\right)\right), \quad k \in \mathcal{G}, \\
& \sum_{m \in \mathcal{N}(k)}\left(W_{k k}\left(t^{\prime}\right)-W_{k m}\left(t^{\prime}\right)\right) y_{k m}^{*}= \\
& -P_{l_{k}}\left(t^{\prime}\right)-j Q_{l_{k}}\left(t^{\prime}\right), k \notin \mathcal{G}, \\
& \underline{V}_{k}^{2} \leq W_{k k}\left(t^{\prime}\right) \leq \bar{V}_{k}^{2}, \quad k \in \mathcal{N}, \\
& \Im\left(W_{k m}\left(t^{\prime}\right)\right) \leq \Re\left(W_{k m}\left(t^{\prime}\right)\right) \tan \left(\theta_{k m}^{\max }\right),(k, m) \in \mathcal{L}, \\
& \left|\left(W_{k k}\left(t^{\prime}\right)-W_{k m}\left(t^{\prime}\right)\right) y_{k m}^{*}\right| \leq S_{k m} \\
& W\left(t^{\prime}\right) \succeq 0, \\
& \operatorname{rank}\left(W\left(t^{\prime}\right)\right)=1 \text {. }
\end{aligned}
$$

Instead of (14), which is difficult due to multiple nonconvex matrix rank-one constraints in $(14 \mathrm{~h})$, we solve its semi-definite relaxation $(\mathrm{SDR})$

$$
\min _{W\left(t^{\prime}\right), R\left(t^{\prime}\right), P_{k_{n}}\left(t^{\prime}\right)} F_{[t, \Psi(t)]} \quad \text { s.t. }(14 b)-(14 h) .
$$

Suppose that $\hat{W}\left(t^{\prime}\right)$ and $\left(\hat{R}\left(t^{\prime}\right), \hat{P}_{k_{n}}\left(t^{\prime}\right)\right), t^{\prime} \in[t, \Psi(t)]$ are the optimal solution of (15). If $\operatorname{rank}\left(W\left(t^{\prime}\right)\right) \equiv 1, t^{\prime} \in[t, \Psi(t)]$, 
then $\hat{V}\left(t^{\prime}\right)$ such that $\hat{W}\left(t^{\prime}\right)=\hat{V}\left(t^{\prime}\right) \hat{V}^{H}\left(t^{\prime}\right)$ together with $\hat{R}\left(t^{\prime}\right)$ and $\hat{P}_{k_{n}}\left(t^{\prime}\right)$ constitute the optimal solution of the nonconvex optimization problem (13). Otherwise, we consider the following problem:

$$
\begin{array}{r}
\left.\min _{W(t), R(t)} F\left(P_{g}(t)\right)\right):=\sum_{k \in \mathcal{G}} f\left(P_{g_{k}}(t)\right) \\
\text { s.t. } \quad(3)-(4),(7),(14 d)-(14 h) \text { for } t^{\prime}=t, \\
\sum_{m \in \mathcal{N}(k)}\left(W_{k k}(t)-W_{k m}(t)\right) y_{k m}^{*}=\left(P_{g_{k}}(t)-P_{l_{k}}(t)\right. \\
\left.-\sum_{k_{n} \in C(t)} \hat{P}_{k_{n}}(t)\right)+j\left(Q_{g_{k}}(t)-Q_{l_{k}}(t)\right), \quad k \in \mathcal{G}, \\
\operatorname{rank}(W(t))=1 .
\end{array}
$$

Note that in contrast to (14) involving $\Psi(t)-t$ matrix variables $W\left(t^{\prime}\right), t^{\prime} \in[t, \Psi(t)]$ and also variables $P_{k_{n}}\left(t^{\prime}\right), k_{n} \in C(t)$ and $t^{\prime} \in[t, \Psi(t)]$, there is only single matrix variable $W(t)$ in (16). The power generation variable $R(t)$ in (16) is latent as it is inferred from $W(t)$ in equation (16c).

Following our previous works [13], [20]-[23], a nonsmooth optimization algorithm (NOA) is proposed to deal with the discontinuous matrix rank-one constraint (16d) in the optimization problem (16). Under condition (14h) in (16b),

$$
\operatorname{Trace}(W(t))-\lambda_{\max }(W(t)) \geq 0,
$$

where $\lambda_{\max }(W(t))$ stands for the maximal eigenvalue of $W(t)$. The discontinuous matrix rank-one constraint (16d) is then equivalently expressed by the following continuous spectral constraint:

$$
\operatorname{Trace}(W(t))-\lambda_{\max }(W(t))=0,
$$

because it means that $W(t)$ has only one nonzero eigenvalue. Thus the quantity $\operatorname{Trace}(W(t))-\lambda_{\max }(W(t))$ expresses the degree of the matrix rank-one constraint satisfaction (17), which is incorporated into the objective (16a), leading to the following penalized optimization problem:

$$
\begin{array}{r}
\min _{W(t), R(t)} F_{\mu}\left(W(t), P_{g}(t)\right):=F\left(P_{g}(t)\right) \\
+\mu\left(\operatorname{Trace}(W(t))-\lambda_{\max }(W(t))\right) \\
\text { s.t. } \quad(16 b)-(16 c),
\end{array}
$$

where $\mu>0$ is a penalty parameter. The above penalized optimization is exact because the constraint (16b) can be satisfied by a minimizer of (18) with a finite value of $\mu$. On the other hand, any $W(t)$ feasible for (18) is also feasible for (16), implying that the optimal value of (18) for any $\mu>0$ is upper bounded by the optimal value of (16).

For any $W^{(\kappa)}(t)$ feasible for the convex constraints (16c)(16b), let $w_{\max }^{(\kappa)}(t)$ be the normalized eigenvector corresponding to the eigenvalue $\lambda_{\max }\left(W^{(\kappa)}(t)\right)$. Then

$$
\begin{aligned}
\lambda_{\max }(W(t)) & =\max _{\|w\|^{2}=1} w^{H} W(t) w \\
& \geq\left(w_{\max }^{(\kappa)}(t)\right)^{H} W(t) w_{\max }^{(\kappa)}(t),
\end{aligned}
$$

i.e. the function $\lambda_{\max }(W(t))$ is lower bounded by the linear function $\left(w_{\max }^{(\kappa)}(t)\right)^{H} W(t) w_{\max }^{(\kappa)}(t)$. Accordingly, the following semi-definite program (SDP) provides an upper bound for

\begin{aligned} & \hline Algorithm 1 NOA 1 for solving $(16) \\ &$\hline 1: Set $\kappa=0$ and $\left(W^{(0)}(t), R^{(0)}(t)\right)=(\hat{W}(t), \hat{R}(t)) \\ &$. 2: Until Trace $\left(W^{(\kappa)}(t)\right)-\left(w_{\max }^{(\kappa)}(t)\right)^{H} W^{(\kappa)}(t) w_{\max }^{(\kappa)}(t) \leq \epsilon \\ &$, solve $(20)$, to find the optimal solution \\ & \\ &$\left(W^{(\kappa+1)}(t), R^{(\kappa+1)}(t)\right)$ and reset $\kappa+1 \rightarrow \kappa \\ &$. 3: Accept $\left(W^{(\kappa)}(t), R^{(\kappa)}(t)\right)$ as the optimal solution of the \\ & nonconvex optimization problem $(16)\end{aligned}$.

the nonconvex optimization problem (18):

$$
\begin{array}{r}
\min _{W(t), R(t)} F_{\mu}^{(\kappa)}(W(t), R(t)):=F\left(P_{g}(t)\right) \\
+\mu\left(\operatorname{Trace}(W(t))-\left(w_{\max }^{(\kappa)}(t)\right)^{H} W(t) w_{\max }^{(\kappa)}(t)\right) \\
\text { s.t. } \quad(16 b)-(16 c),
\end{array}
$$

because

$$
F_{\mu}^{(\kappa)}(W(t), R(t)) \geq F_{\mu}(W(t), R(t))
$$

according to (19).

Suppose that $\left(W^{(\kappa+1)}(t), R^{(\kappa+1)}(t)\right)$ is the optimal solution of SDP (20). Since $\left(W^{(\kappa)}(t), R^{(\kappa)}(t)\right)$ is also feasible for (20), it is true that

$$
\begin{aligned}
& F_{\mu}\left(W^{(\kappa)}(t), R^{(\kappa)}(t)\right) \\
= & F_{\mu}^{(\kappa)}\left(W^{(\kappa)}(t), R^{(\kappa)}(t)\right) \\
\geq & F_{\mu}^{(\kappa)}\left(W^{(\kappa+1)}(t), R^{(\kappa)+1}(t)\right) \\
\geq & F_{\mu}\left(W^{(\kappa+1)}(t), R^{(\kappa+1)}(t)\right),
\end{aligned}
$$

so $W^{(\kappa+1)}(t)$ is a better feasible point of (18) than $W^{(\kappa)}(t)$.

In Nonsmooth Optimization Algorithm (NOA) 1 we propose an iterative procedure, which is initialized by the solution $\hat{W}(t)$ of SDR (15) and generates a feasible point $W^{(\kappa+1)}(t)$ at the $\kappa$-th iteration for $\kappa=0,1, \ldots$, as the optimal solution of SDP (18). As proved in [13], this algorithm converges at least to a local minimizer of (18). Note that the procedure terminates at

$$
\begin{aligned}
0 & \leq \operatorname{Trace}\left(W^{(\kappa)}(t)\right)-\lambda_{\max }\left(W^{(\kappa)}(t)\right) \\
& \leq \operatorname{Trace}\left(W^{(\kappa)}(t)\right)-\left(w_{\max }^{(\kappa)}(t)\right)^{H} W^{(\kappa)}(t) w_{\max }^{(\kappa)}(t) \\
& \leq \epsilon,
\end{aligned}
$$

so the spectral constraint (17) for the matrix rank-one is satisfied with the computational tolerance $\epsilon$. In summary, our proposed MPC-based computation for (11) is based on solving SDP (15) for online coordinating PEV charge $\hat{P}_{k_{n}}(t)$ and solving (18) by NOA 1 for online updating the generated voltage $\hat{V}(t)$ for the generated power $\hat{R}(t)$ by

$$
\hat{V}(t)=\sqrt{\lambda_{\max }\left(W^{(\kappa)}\right)} w_{\max }^{(\kappa)}(t),
$$

whenever the solution $\hat{W}(t)$ of SDR (15) is not of rankone. If $\operatorname{rank}(\hat{W}(t))=1$, it is obvious that $\hat{V}(t)=$ $\sqrt{\lambda_{\max }(\hat{W}(t))} \hat{w}_{\max }(t)$ with the normalized eigenvector $\hat{w}_{\max }(t)$ corresponding to $\lambda_{\max }(\hat{W}(t))$ is the optimal solution of (13), which is what we need. 


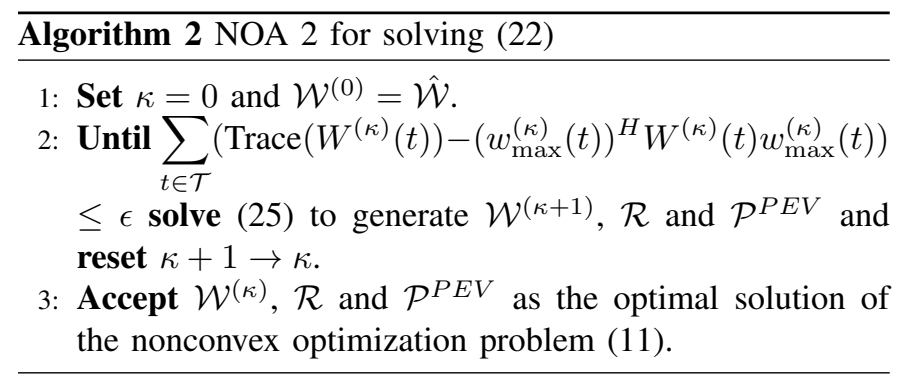

\section{LOWER BOUND BY OFF-LINE OPTIMIZATION}

To investigate the optimality of the MPC-based online computation proposed in the previous section, in this section we address an off-line computation for (11), which provides a lower bound for the optimal value of its online computation. Under the additional definition $\mathcal{W}=\left\{W\left(t^{\prime}\right)\right\}_{t^{\prime} \in \mathcal{T}}$, we reformulate (11) as

$$
\begin{array}{r}
\min _{\mathcal{W}, \mathcal{R}, \mathcal{P}^{P E V}} F\left(\mathcal{R}, \mathcal{R}^{P E V}\right) \quad \text { s.t. (22a) } \\
(2)-(4),(7),(14 d)-(14 h) \text { for } t^{\prime} \in \mathcal{T},(22 \mathrm{~b}) \\
\sum_{m \in \mathcal{N}(k)}\left(W_{k k}\left(t^{\prime}\right)-W_{k m}\left(t^{\prime}\right)\right) y_{k m}^{*}=\left(P_{g_{k}}\left(t^{\prime}\right)-P_{l_{k}}\left(t^{\prime}\right)\right. \\
\left.-\sum_{n \in \mathcal{H}_{k}} P_{k_{n}}\left(t^{\prime}\right)\right)+j\left(Q_{g_{k}}\left(t^{\prime}\right)-Q_{l_{k}}\left(t^{\prime}\right)\right), k \in \mathcal{G},(22 \mathrm{c}) \\
\operatorname{rank}\left(W\left(t^{\prime}\right)\right)=1, t^{\prime} \in \mathcal{T} .(22 \mathrm{~d})
\end{array}
$$

First, we solve its SDR by dropping the matrix rank-one constraints in $(22 \mathrm{~d})$ :

$$
\min _{\mathcal{W}, \mathcal{R}^{P}, \mathcal{P}^{P E V}} F\left(\mathcal{R}, \mathcal{R}^{P E V}\right) \quad \text { s.t. } \quad(22 b)-(22 c) .
$$

Suppose that $\hat{\mathcal{W}}$ and $\hat{\mathcal{P}}^{P E V}$ are the optimal solution of SDP (23). If $\operatorname{rank}(\hat{W}(t)) \equiv 1, t \in \mathcal{T}$ then a global solution of the original problem (11) is found as $\hat{\mathcal{P}}^{P E V}, \hat{\mathcal{R}}$ and $\hat{\mathcal{V}}$ and with $\hat{V}(t) \hat{V}^{H}(t)=\hat{W}(t), t \in \mathcal{T}$. However, such a matrix rank-one condition is rarely achieved. In what follows we propose two methods to address the matrix rank-one constraints in (22d).

Again, under condition (14h) for $t^{\prime} \in \mathcal{T}$ in (22b), the rankone constraints in (22d) are equivalently expressed by the single spectral constraint $\sum_{t \in \mathcal{T}}\left(\operatorname{Trace}(W(t))-\lambda_{\max }(W(t))\right)=$ 0 , which is incorporated into the objective function in (22a) for the following penalized function optimization:

$$
\begin{array}{r}
\min _{\mathcal{W}, \mathcal{R}, \mathcal{P} P E V} F\left(\mathcal{R}, \mathcal{R}^{P E V}\right)+\mu \sum_{t \in \mathcal{T}}(\operatorname{Trace}(W(t))- \\
\left.\lambda_{\max }(W(t))\right) \quad \text { s.t. } \quad(22 b)-(22 c),
\end{array}
$$

with a penalty parameter $\mu>0$. Initialized by $\mathcal{W}^{(0)}=\hat{\mathcal{W}}$, the following SDP is solved at $\kappa$-th iteration to generate $\mathcal{W}^{(\kappa+1)}$ and $\mathcal{P}^{P E V}$ :

$$
\begin{gathered}
\min _{\mathcal{W}, \mathcal{R}, \mathcal{P}^{P E V}} F\left(\mathcal{R}, \mathcal{R}^{P E V}\right)+\mu \sum_{t \in \mathcal{T}}(\operatorname{Trace}(W(t))- \\
\left.\left(w_{\max }^{(\kappa)}(t)\right)^{H} W(t) w_{\max }^{(\kappa)}(t)\right) \quad \text { s.t. } \quad(22 b)-(22 c) .
\end{gathered}
$$

This computational procedure is summarized in NOA 2.

Alternatively, we propose the following scalable algorithm for computing (22). By replacing $P_{k_{n}}(t)$ by $\hat{P}_{k_{n}}(t)$, which

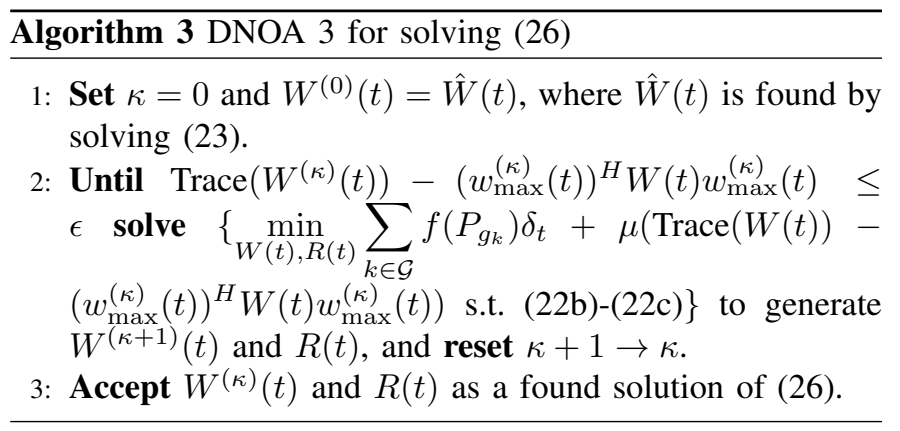

was found by solving from (23), in (22) at every $t \in \mathcal{T}$, we obtain the following optimization problem in $W(t)$ and $R(t)$ only:

$$
\begin{array}{r}
\min _{W(t), R(t)} \sum_{k \in \mathcal{G}} f\left(P_{g_{k}}\right) \text { s.t. }(22 b)-(22 c) \text { for } t^{\prime}=t, \\
\operatorname{rank}(W(t))=1,
\end{array}
$$

which is computed by the distributed NOA Algorithm (DNOA) 3.

\section{Simulation Results}

\section{A. Simulation setup}

The energy sources of the charging stations come from the transmission stations or from self-generated sources such as photovoltaics. Without loss of generality, the charging stations are set at generator buses, which however are not necessarily far from the residential neighborhood. The proposed method should be conducted by Distribution System Operators (DSOs) and still works whenever the charging stations are set at other buses in the grid. Roughly speaking, DSOs are divided into three segments in terms of customer care [24]: DSOs connected to the transmission system, regional DSOs and local DSOs. Like [12], DSOs in our set-up thus belongs to the first segment, where the CSs and PEVs serve as distribution systems connected to transmission networks. Each CS broadcasts the charging commands and communicates the charging demands with connected PEVs.

The SDPs (15), (20), (23) and (25) are computed using Sedumi [25] interfaced by CVX [26] on a Core i5-3470 processor. Four power networks from Matpower [27] are chosen. The tolerance $\epsilon=10^{-4}$ is set for the stop criterions.

Generally, PEVs are charged after their owners' working hours. We focus on the charging period from 6:00 pm to 6:00 am of the next day, which is then uniformly divided into 24 time slots of 30 minute length [28]. Accordingly, the charging time horizon is $\mathcal{T}=\{1,2, \ldots, 24\}$. It is also reasonable to assume that the PEVs arrive during the time period from 6:00 pm to midnight. The PEVs must be fully charged after being plugged into the grid. During this time period, PEV charging demands usually have one peak [29, Fig. 3], [30, Fig. 2] and [31, Fig 3], so the arrival times of PEVs are assumed to be independent and can be generated by a truncated normal distribution $\left(20,1.5^{2}\right)$, which is depicted by Fig. 2 .

We assume that the PEVs are Tesla Model S's, which have a battery capacity of $100 \mathrm{KWh}$ [32]. The SOC of all PEVs is 


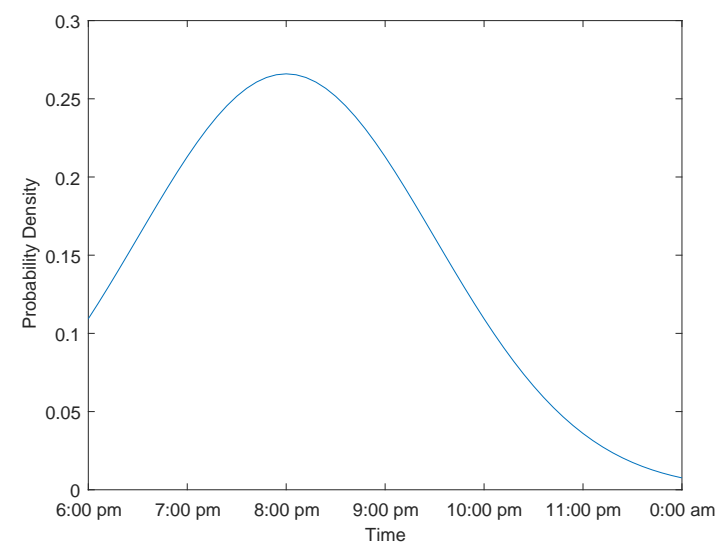

Fig. 2: The probability density of PEVs' arrivals

set as $20 \%$. The structure and physical limits of the considered grids are given in the Matpower library [27] together with the specific cost functions $f\left(P_{g_{k}}(t)\right)$.

The price-inelastic load $P_{l_{k}}(t)$ is calculated as

$$
P_{l_{k}}(t)=\frac{l(t) \times \bar{P}_{l_{k}} \times T}{\sum_{t=1}^{24} l(t)}, \quad t \in \mathcal{T},
$$

where $\bar{P}_{l_{k}}$ is the load demand specified by [27] and $l(t)$ is the residential load demand taken from [33]. Four profiles are taken from different days in 2017 [33]. Profile 1 is the residential load and energy price from 6:00 pm on February 5 th to 6:00 am on February 6th, Profile 2 is from 6:00 pm on March 5th to 6:00 am on March 6th, Profile 3 is from 6:00 pm on April 5th to 6:00 am on April 6th, and Profile 4 is from 6:00 pm on May 5th to 6:00 am on May 6th. Fig. 3 and Fig. 4 provide the residential load demand and energy price for these profiles.

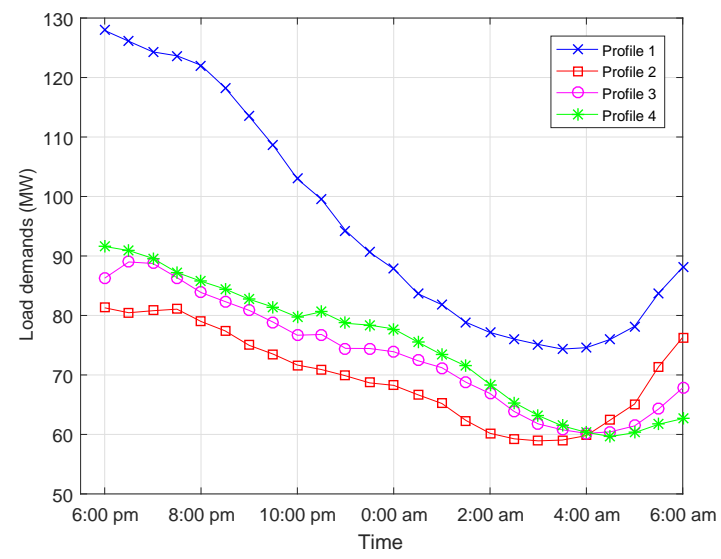

Fig. 3: Residential load demands of four profiles

\section{B. MPC-based online computational results}

1) Four network simulation: We test MPC-based online computation for Case9, Case14, Case30 and Case118mod from [27] and profile 2 of the residential data. The information on these networks is given in Table. I, where the first column is the name of network, the second column indicates the numbers of buses, generators and branches. The dimension of

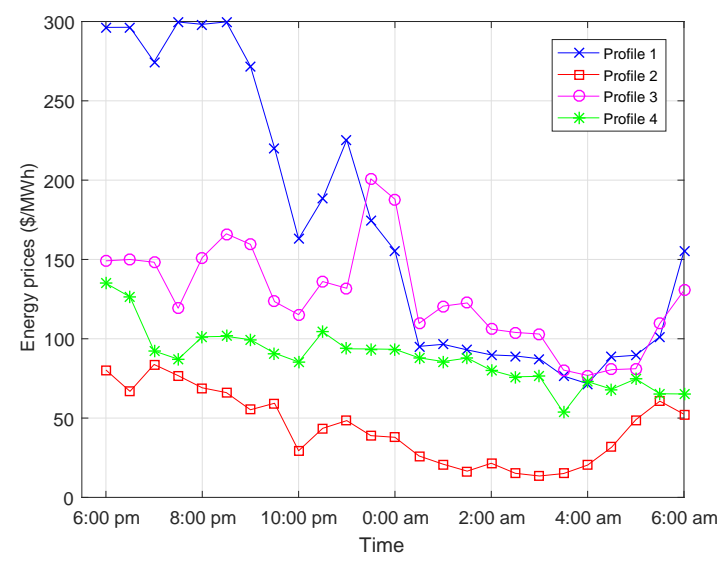

Fig. 4: Energy prices for four profiles

$W(t)$ is given in the third column, while the total number of PEVs is shown in the last column. The computational results

TABLE I: Information on four networks

\begin{tabular}{cccc}
\hline & Buses/Generators/Branches & Dim. of $W(t)$ & PEVs \\
\hline Case9 & $9 / 3 / 9$ & $\mathbb{C}^{9 \times 9}$ & 291 \\
Case14 & $14 / 5 / 20$ & $\mathbb{C}^{14 \times 14}$ & 485 \\
Case30 & $30 / 6 / 24$ & $\mathbb{C}^{30 \times 30}$ & 582 \\
Case118mod & $118 / 54 / 186$ & $\mathbb{C}^{118 \times 118}$ & 5238 \\
\hline
\end{tabular}

are summarized in Table II. Again, the first column is the

TABLE II: MPC results

\begin{tabular}{cccccc}
\hline & Rank & $\mu$ & LB & Comp.value & Time(s) \\
\hline Case9 & 9 & 10 & 27991.4 & 27992.3 & 7.4 \\
Case14 & 1 & - & 40824.1 & 40824.1 & 8.5 \\
Case30 & 1 & - & 4935.6 & 4935.6 & 8.7 \\
Case118mod & 2 & 100 & 644245.9 & 644278.5 & 432.1 \\
\hline
\end{tabular}

network name. The second column presents the initial rank of the optimal solution $\hat{W}(t)$ of SDR (15). It is observed that the rank of $\hat{W}(t)$ is the same for all $t \in \mathcal{T}$. The value of the penalty parameter $\mu$ in (18) is given in the third column. As the initial rank of Case14 and Case 30 are all rank-one, SDR (15) already outputs the optimal solution for (13). Comparing the lower bound (LB) in the fourth column by solving SDR (23) at each time and the value found by the proposed MPC-based computation with using NOA 1 in computing (11) in each time reveals the capability of the MPC-based computation for (11). These values are either the same (for Case14 and Case30) or almost the same (for Case 9 and Case118mod), so indeed the proposed MPC-based computation could exactly locate a globally optimal solution. The average running time for solving (13) to implement the proposed MPC-based computation is provided in the sixth column, which is very short compared with the 30 minute time slot and thus is practical for this particular online application.

The voltage profile for the four networks during the charging period are shown in Fig. 5. For all cases, the voltage bound constraints (8) are satisfied. The voltage behavior is stable and smooth.

2) Four residential profile simulation: We consider Case 30 together with four different residential profiles. The computa- 

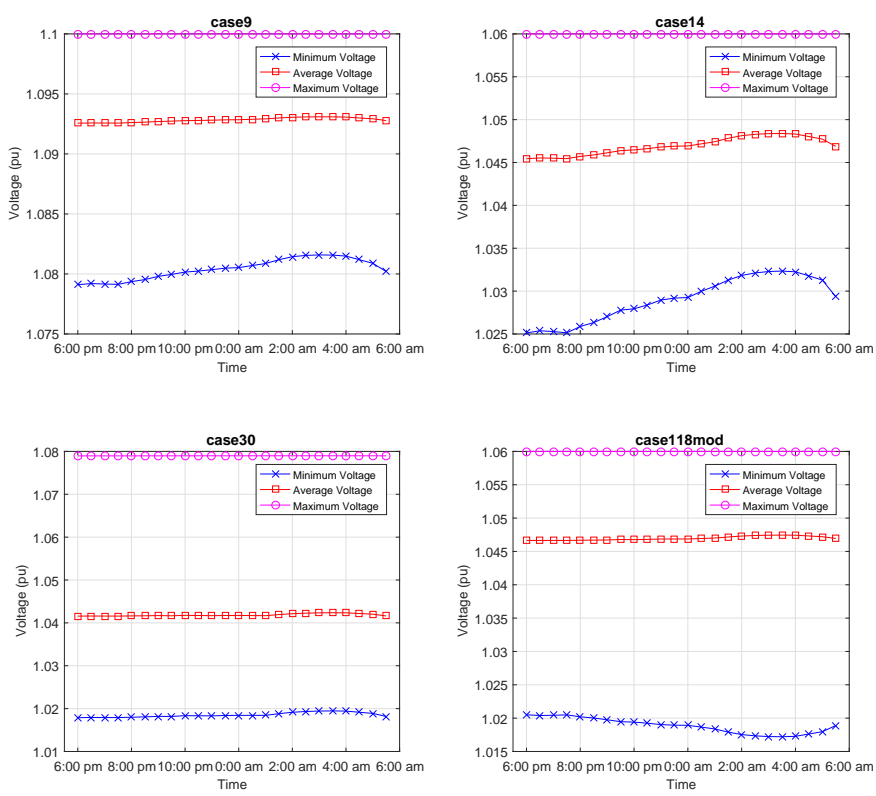

Fig. 5: Voltage profile for four networks during the charging period

tional results are provided in Table III, whose format is similar to Table II. It can be seen that, even for the same network,

TABLE III: MPC results for Case30 with four different residential profiles

\begin{tabular}{cccccc}
\hline & Rank & $\mu$ & LB & Comp. value & Time(s) \\
\hline Profile 1 & 2 & 10 & 31961.2 & 31963.5 & 10.9 \\
Profile 2 & 1 & - & 4963.3 & 4963.3 & 8.7 \\
Profile 3 & 2 & 10 & 10771.3 & 10774.7 & 8.7 \\
Profile 4 & 1 & - & 8139.3 & 8139.3 & 8.1 \\
\hline
\end{tabular}

the rank of the optimal solution $\hat{W}(t)$ of SDR (15) may be different depending on the residential profiles. For profile 2 and profile 4, the initial rank is one and SDR (15) has located a globally optimal solution. However, for profile 1 and profile 3 , NOA 1 is needed for obtaining the rank-one solution. The convergence speed is fast, and the optimum values are all equal or close to the lower bound, which clearly shows the global efficiency of the proposed MPC-based computation.

The aggregated active powers generated at each time are shown in Fig. 6, from which the trends of generated power are seen to be similar to the residential load demand in Fig. 3.

The stable and smooth voltage profile for these 4 residential profiles during the charging period are shown in Fig. 7.

Fig. 8 plots the $\mathrm{SoC}$ of four PEVs randomly taken from case 30 under profile 2, which arrive at different times.

\section{Off-line computation and comparison with MPC-based online computation}

Firstly, Case9, Case14, Case30 and Case118mod are tested with the residential data of profile 2 to analyze the efficiency of off-line computation by using Algorithm 2 and Algorithm 3. The computational results are summarized in Table IV. The initial rank in the second column is the rank of the

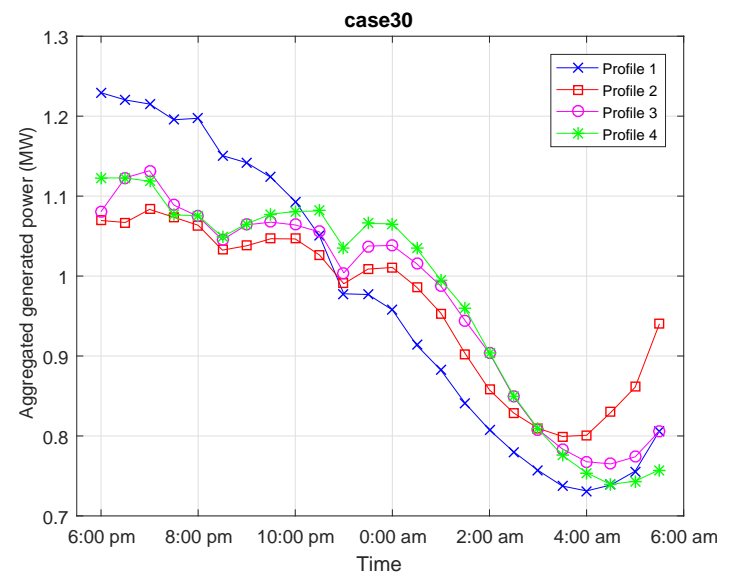

Fig. 6: Aggregated active power of online charging for Case30 under four residential profiles
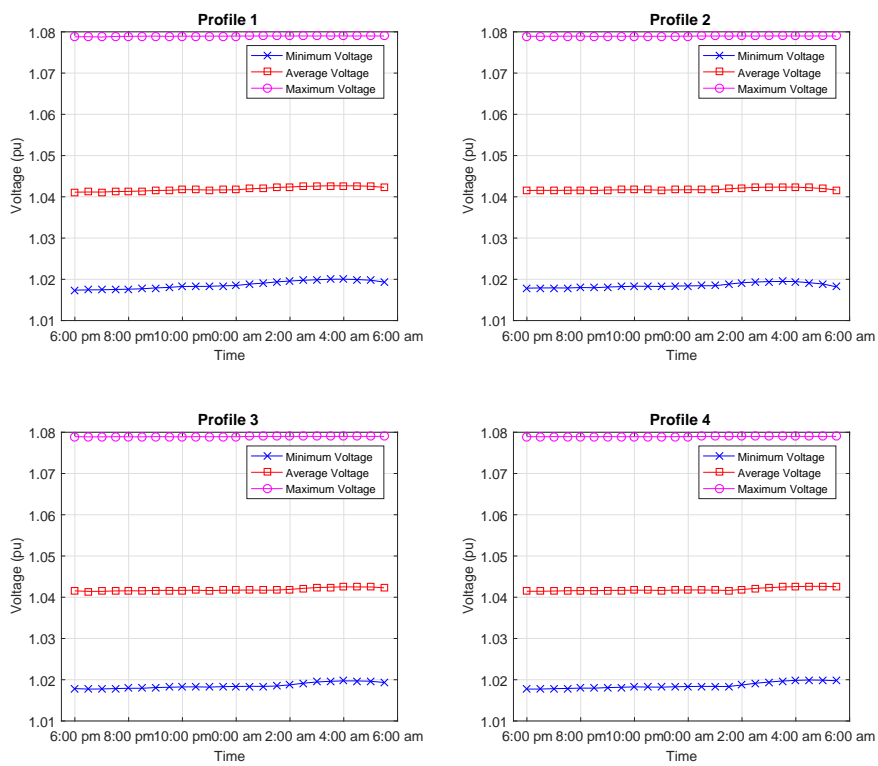

Fig. 7: Voltage profile of online charging for Case30 under four residential profiles

optimal solution $\hat{W}(t)$ of SDR (23), which is the same for all $t \in \mathcal{T}$. The value of penalty parameter $\mu$ in (25) is in the third column. The fourth column provides the lower bound by computing SDR (23). The values found by solving (24) and (26) by Algorithm 2 and Algorithm 3 are in the fifth column as they are the same and are either exactly same as their lower bounds in the fourth column (Case9, Case14 and Case30) or almost the same (Case118mod). According to the seventh column both Algorithm 2 and Algorithm 3 converge in two and three iterations for Case 9 and Case $188 \mathrm{mod}$, while for Case9 and Case30, SDR (23) already outputs the optimal rank-one solution. The running times of Algorithm 2 and Algorithm 3 are provided in the eighth and ninth column, respectively. Algorithm 2 requires less running time for smallscale networks such as Case9, Case14 and Case118mod. However, its running time increases dramatically for largescale networks such as Case118mod, for which the scalable Algorithm 3 is clearly advantageous. 
TABLE IV: Offline results of optimal PEV charging for four networks

\begin{tabular}{ccccccccc}
\hline & Rank & $\mu$ & Lower bound & Computed value & Opt. degree & Iterations & NOA time(s) & DNOA time(s) \\
\hline Case9 & 9 & 1 & 27978.1 & 27978.1 & $100 \%$ & 2 & 11.2 & 23.2 \\
Case14 & 1 & - & 40800.7 & 40800.7 & $100 \%$ & 1 & 8.9 & 8.9 \\
Case30 & 1 & - & 4935.6 & 4935.6 & $100 \%$ & 1 & 24.5 & 36.3 \\
Case118mod & 2 & 50 & 644225.3 & 644233.9 & $99.999 \%$ & 3 & 1094.8 & 363.5 \\
\hline
\end{tabular}

TABLE V: Performance comparison under MPC-based and off-line computations

\begin{tabular}{llcccccc}
\hline & & Rank & MPC & Offline & Offline/MPC & MPC times(s) & Offline times(s) \\
\hline \multirow{5}{*}{ Case9 } & Profile 1 & 9 & 31963.5 & 31963.1 & $99.99 \%$ & 161.1 & 15.2 \\
& Profile 2 & 9 & 27992.3 & 27978.1 & $99.94 \%$ & 177.4 & 15.1 \\
& Profile 3 & 9 & 31102.9 & 30885.1 & $99.29 \%$ & 173.7 & 14.8 \\
& Profile 4 & 9 & 29896.2 & 29870.9 & $99.91 \%$ & 178.4 & 15.0 \\
\hline \multirow{5}{*}{ Case30 } & Profile 1 & 2 & 31963.5 & 31963.1 & $99.99 \%$ & 262.4 & 37.4 \\
& Profile 2 & 1 & 4963.3 & 4935.6 & $99.43 \%$ & 209.8 & 12.6 \\
& Profile 3 & 2 & 10774.7 & 10330.8 & $95.70 \%$ & 208.2 & 24.5 \\
& Profile 4 & 1 & 8139.3 & 8087.2 & $99.35 \%$ & 194.9 & 12.9
\end{tabular}

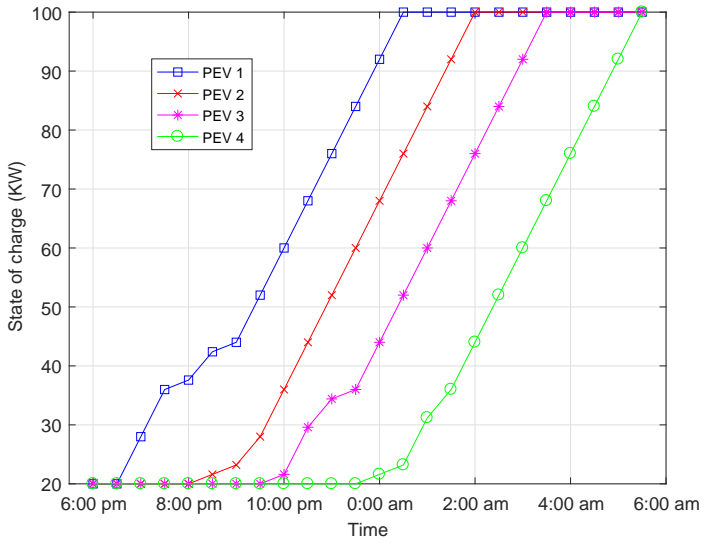

Fig. 8: The SOC of PEVs during the charging period

A performance comparison between MPC-based computation and off-line computation for Case 9 and Case 30 with the four mentioned residential profiles is provided in Table V, which clearly shows the global optimality of the proposed MPC-based computation as it attains objective values very close to the lower bounds provided by the off-line computation.

Fig. 9 plots online and offline power generations in Case 30 with four residential profiles, while Fig. 10 plots the corresponding PEV charging scheduling. The charging load drops dramatically after $0: 00 \mathrm{am}$, by which all PEVs have been integrated into the grid but some of them have already been fully charged. Obviously, the charging load is sensitive to the energy price. For example in profile 3, the increase of the energy price at $11: 30 \mathrm{pm}$ and $0: 00 \mathrm{am}$ leads to a significant drop of the charging load. It should be noted that, energy price is not the only impact factor for the aggregating charging rate in Fig. 10. The power balance and residential demands also have significant effects on the aggregating charging rate. From 6:00 pm to 9:00 pm PEVs are continuingly connected to the grid, so the aggregating charging rate during that time is increased. The charging load under MPC-based and off-line simulation are the same after $0: 00$ am because there are no new PEVs arriving after that time.

Fig. 11 presents the SoC of four PEVs randomly taken from
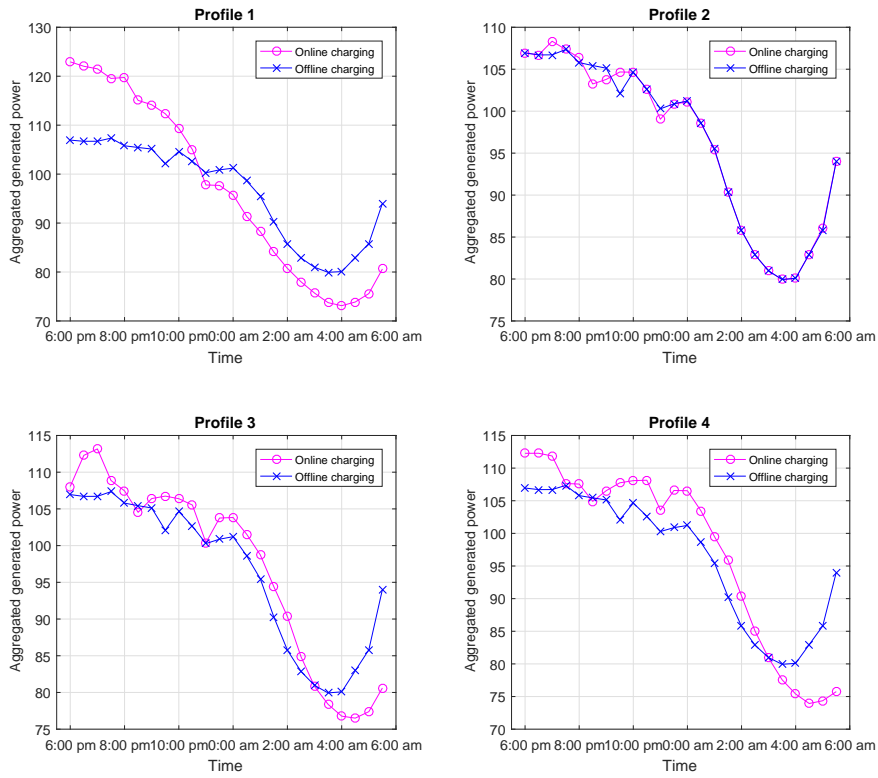

Fig. 9: Power generation under MPC-based (online) and offline computation for Case30 with four residential profiles

Case30 with profile 2. It can be seen that, though the objective values shown in Table. V look similar, the SoC are different by online and offline algorithms.

\section{CONCLUSIONS}

Joint PEV charging scheduling and power control for power grids to serve both PEVs at a competitive cost and residential power demands at a competitive operating cost is very difficult due to the random nature of PEVs' arrivals and demands. We have proposed a novel and easily-implemented MPC-based computational algorithm that can achieve a globally optimal solution.

\section{REFERENCES}

[1] W. Tusha, C. Yuen, S. Huang, D. B. Smith, and H. V. Poor, "Cost minimization of charging stations with photovoltaics: An approach with EV classification," IEEE Trans. Intell. Transport. Systs., vol. 17, no. 1, pp. 156-169, 2016. 

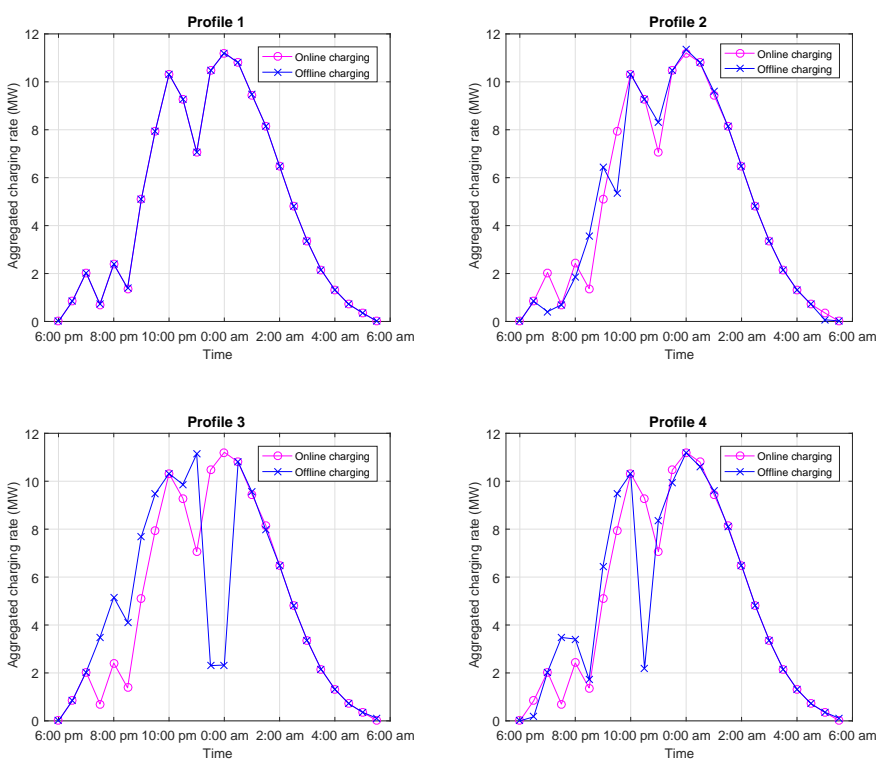

Fig. 10: PEVs charging load under MPC-based and offline computation for Case 30 with four residential profiles
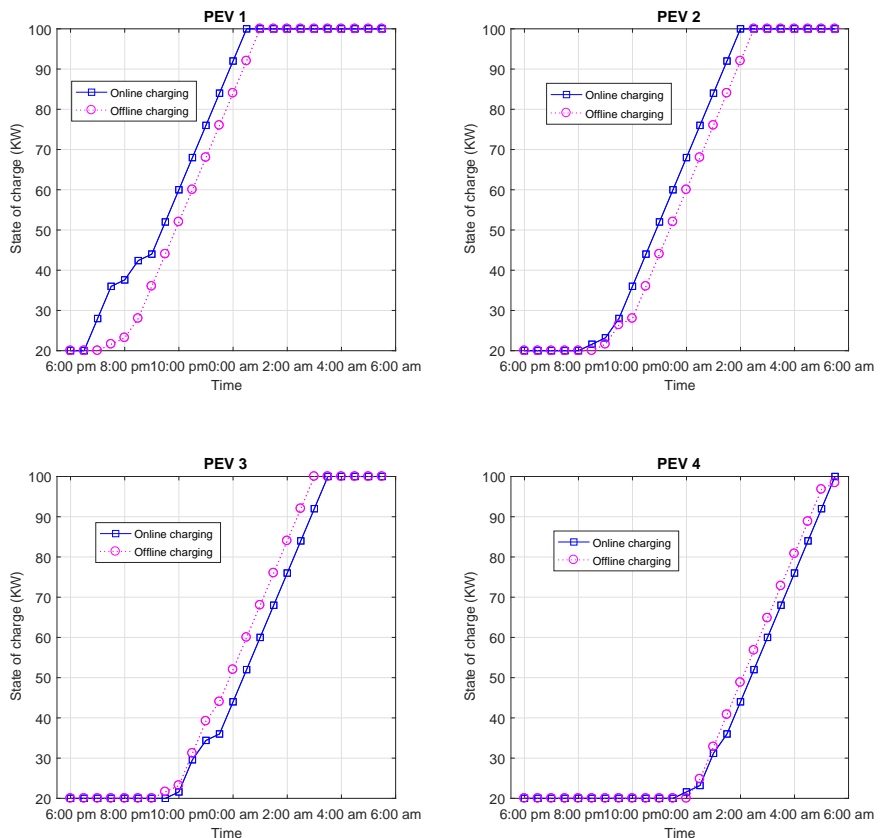

Fig. 11: SOC of PEVs randomly taken from Case30 with profile 2

[2] W. Tang, S. Bi, and Y. J. Zhang, "Online charging scheduling algorithms of electric vehicles in smart grid: An overview," IEEE Commun. Mag., vol. 54, no. 12, pp. 76-83, 2016.

[3] Y. Wang, W. Saad, Z. Han, H. V. Poor, and T. Basar, "A game-theoretic approach to energy trading in the smart grid," IEEE Trans. Smart Grid, vol. 5, no. 3, pp. 1439-1450, 2014.

[4] L. Yang, J. Zhang, and H. V. Poor, "Risk-aware day-ahead scheduling and real-time dispatch for electric vehicle charging," IEEE Trans. Smart Grid, vol. 5, no. 2, pp. 693-702, 2014.

[5] Y. Wang, W. Saad, N. B. Mandayam, and H. V. Poor, "Load shifting in the smart grid: To participate or not?," IEEE Trans. Smart Grid, vol. 7 , no. 6, pp. 2604-2614, 2016.

[6] S. Lakshminarayana, Y. Xu, H. V. Poor, and T. Q. S. Quek, "Cooperation of storage operation in a power network with renewable generation," IEEE Trans. Smart Grid, vol. 7, no. 4, pp. 2108-2122, 2016.
[7] G. Li and X.-P. Zhang, "Modeling of plug-in hybrid electric vehicle charging demand in probabilistic power flow calculations," IEEE Trans. Smart Grid, vol. 3, no. 1, pp. 492-499, 2012.

[8] H. Mohsenian-Rad and M. Ghamkhari, "Optimal charging of electric vehicles with uncertain departure times: A closed-form solution," IEEE Trans. Smart Grid, vol. 6, no. 2, pp. 940-942, 2015.

[9] H. Xing, M. Fu, Z. Lin, and Y. Mou, "Decentralized optimal scheduling for charging and discharging of plug-in electric vehicles in smart grids," IEEE Trans. Power Syst., vol. 31, no. 5, pp. 4118-4127, 2016.

[10] W. Tang and Y. J. A. Zhang, "A model predictive control approach for low-complexity electric vehicle charging scheduling: optimality and scalability," IEEE Trans. Power Systems, vol. 32, no. 2, pp. 1050-1063, 2017.

[11] Y. Kim, J. Kwak, and S. Chong, "Dynamic pricing, scheduling, and energy management for profit maximization in PHEV charging stations," IEEE Trans. Vehic. Tech., vol. 66, no. 2, pp. 1011-1026, 2017.

[12] N. Chen, C. W. Tan, and T. Q. Quek, "Electric vehicle charging in smart grid: Optimality and valley-filling algorithms," IEEE J. Sel.Topics Signal Process., vol. 8, no. 6, pp. 1073-1083, 2014.

[13] Y. Shi, H. D. Tuan, H. Tuy, and S. Su, "Global optimization for optimal power flow over transmission networks," J. Global Optimz. (to appear), 2017.

[14] J. F. Franco, M. J. Rider, and R. Romero, "A mixed-integer linear programming model for the electric vehicle charging coordination problem in unbalanced electrical distribution systems," IEEE Trans. Smart Grid, vol. 6, pp. 2200-2210, Sept 2015.

[15] L. Zhang, V. Kekatos, and G. B. Giannakis, "Scalable electric vehicle charging protocols," IEEE Trans. Power Systems, vol. 32, no. 2, pp. 1451-1462, 2017.

[16] E. F. Camacho and C. Bordons, Model Predictive Control. Springer: Springer-Verlag, 2004.

[17] A. Mesbah, "Stochastic model predictive control: An overview and perspectives for future research," IEEE Control Systems Mag., vol. 36, no. 6, pp. 30-44, 2016.

[18] J. W. Polderman and J. C. Willems, Introduction to Mathematical Systems Theory: A Behavioral Approach, 2nd Edition. Springer-Verlag New York, 1998.

[19] H. D. Tuan, A. Savkin, T. Nguyen, and H. T. Nguyen, "Decentralised model predictive control with stability constraints and its application in process control," J. of Process Control, vol. 26, pp. 73-89, 2015.

[20] A. H. Phan, H. D. Tuan, H. H. Kha, and D. T. Ngo, "Nonsmooth optimization for efficient beamforming in cognitive radio multicast transmission," IEEE Trans. Sign. Process., vol. 60, no. 6, pp. 29412951, 2012

[21] Y. Shi, H. D. Tuan, S. W. Su, and H. H. M. Tam, "Nonsmooth optimization for optimal power flow over transmission networks," in 2015 IEEE Global Conf. Signal Info. Process. (GlobalSIP), pp. 11411144, Dec. 2015

[22] A. A. Nasir, H. D. Tuan, D. T. Ngo, T. Q. Duong, and H. V. Poor, "Beamforming design for wireless information and power transfer systems: Receive power-splitting versus transmit time-switching," IEEE Trans. Commun., vol. 65, no. 2, pp. 876-889, 2017.

[23] Y. Shi, H. D. Tuan, and P. Apkarian, "Nonconvex spectral optimization algorithms for reduced-order $\mathcal{H}_{\infty}$ LPV-LFT controllers," Int. J. Nonlinear Robust Control, vol. 27, 2017.

[24] "Distribution system operators." https://www.swissgrid.ch/swissgrid/en/ home/experts/dso.html.

[25] J. Sturm, "Using SeDuMi 1.02, a MATLAB toolbox for optimization over symmetric cones," Optimization Methods and Software, vol. 11-12, pp. 625-653, 1999.

[26] M. Grant and S. Boyd, "CVX: Matlab software for disciplined convex programming, version 2.1." http://cvxr.com/cvx, Mar. 2014.

[27] R. D. Zimmerman, C. E. Murillo-Sanchez, and R. J. Thomas, "Matpower: Steady-state operations, planning, and analysis tools for power systems research and education," IEEE Trans. Power Systems, vol. 26, pp. 12-19, Feb 2011.

[28] C. Jin, J. Tang, and P. Ghosh, "Optimizing electric vehicle charging: A customer's perspective," IEEE Trans. Veh. Tech., vol. 62, pp. 2919-2927, Sept 2013.

[29] J. Zheng, X. Wang, K. Men, C. Zhu, and S. Zhu, "Aggregation modelbased optimization for electric vehicle charging strategy," IEEE Trans. Smart Grid, vol. 4, no. 2, pp. 1058-1066, 2013.

[30] L. Chen, C. Y. Chung, Y. Nie, and R. Yu, "Modeling and optimization of electric vehicle charging load in a parking lot," in 2013 IEEE PES AsiaPacific Power and Energy Engineering Conference (APPEEC), pp. 1-5, Dec 2013. 
[31] C. S. Antnez, J. F. Franco, M. J. Rider, and R. Romero, "A new methodology for the optimal charging coordination of electric vehicles considering vehicle-to-grid technology," IEEE Trans. Sustainable Energy, vol. 7, pp. 596-607, April 2016.

[32] "Tesla model s." https://en.wikipedia.org/wiki/Tesla_Model_S. Accessed: 2017-06-06.

[33] "Australian energy market operator." https://www.aemo.com. au/Electricity/National-Electricity-Market-NEM/Data-dashboard\# price-demand. Accessed: 2017-06-06.

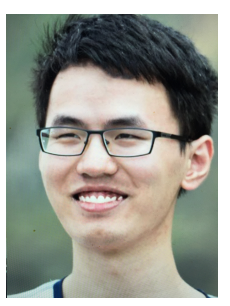

Ye Shi received the B.S. degree in statistics from Northwestern Polytechnical University, Xian, China, in 2013. He is currently working toward the Ph.D. degree at the University of Technology, Sydney, Sydney, Australia. His research interests include nonconvex optimization, smart grid and robust control. He received Best Paper Award at the 6th IEEE International Conference on Control Systems, Computing and Engineering in 2016.

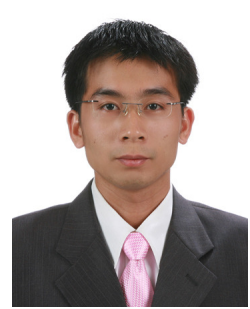

Trung Q. Duong (S'05, M'12, SM'13) received his $\mathrm{Ph} . \mathrm{D}$. degree in Telecommunications Systems from Blekinge Institute of Technology (BTH), Sweden in 2012. Since 2013, he has joined Queen's University Belfast, UK as a Lecturer (Assistant Professor). His current research interests include small-cell networks, physical layer security, energy-harvesting communications, cognitive relay networks. $\mathrm{He}$ is the author or co-author of more than 260 technical papers published in scientific journals (142 articles) and presented at international conferences $(121 \mathrm{pa}-$ pers).

Dr. Duong currently serves as an Editor for the IEEE TRANSACTIONS ON WirEless COMMUNICATIONS, IEEE TRANSACTIONS ON COMMUNICATIONS, IET COMMUNiCATIONS, and a Senior Editor for IEEE COMMUNiCATIONS LetTers. He was awarded the Best Paper Award at the IEEE Vehicular Technology Conference (VTC-Spring) in 2013, IEEE International Conference on Communications (ICC) 2014, and IEEE Global Communications Conference (GLOBECOM) 2016. He is the recipient of prestigious Royal Academy of Engineering Research Fellowship (2016-2021).

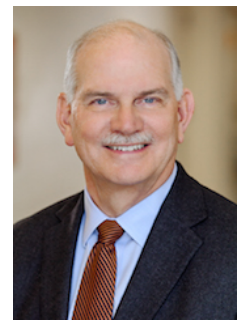

H. Vincent Poor (S72, M77, SM82, F87) received the Ph.D. degree in EECS from Princeton University in 1977. From 1977 until 1990, he was on the faculty of the University of Illinois at UrbanaChampaign. Since 1990 he has been on the faculty at Princeton, where he is currently the Michael Henry Strater University Professor of Electrical Engineering. During 2006 to 2016, he served as Dean of Princetons School of Engineering and Applied Science. Dr. Poors research interests are in the areas of information theory and signal processing, and their applications in wireless networks, energy systems, and related fields. Among his publications in these areas is the recent book Information Theoretic Security and Privacy of Information Systems (Cambridge University Press, 2017).

Dr. Poor is a Member of the National Academy of Engineering and the National Academy of Sciences, and is a Foreign Member of the Chinese Academy of Sciences, the Royal Society, and other national and international academies. Recent recognition of his work includes the 2017 IEEE Alexander Graham Bell Medal, Honorary Professorships at Peking University and Tsinghua University, both conferred in 2016, and a Doctor of Science honoris causa from Syracuse University, awarded in 2017.

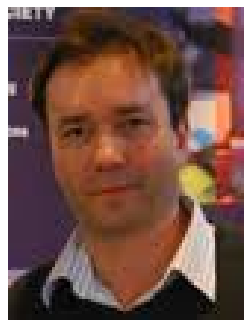

Andrey V. Savkin received MS degree in 1987 and PhD degree in 1991, both from the Leningrad State University. He is a full professor with the School of Electrical Engineering and Telecommunications, the University of New South Wales, Sydney, Australia since 2000. He has authored and co-authored 7 research monograph (published by Springer, Birkhauser, IEEE Press - Wiley and Elsevier) and about 200 journal papers. Prof. Savkin served as an Associate and/or Guest Editor for several international journals and numerous international conferences in the field. His current research interests include applications of control theory to robotics, power systems, sensor networks and biomedical engineering. 